\title{
Twenty strong years with plenty still to do
}

\author{
The Very Large Telescope's Ultraviolet and Visual Echelle Spectrograph (UVES) recently marked 20 years of \\ operations, but the job is not done for this workhorse instrument, write Instrument Scientist Luca Sbordone and \\ Fellow Camila Navarrete.
}

A

fter almost six months closed as the COVID-19 pandemic sent most of Chile into lockdown, on 13 September 2020 the dome of the $8.2 \mathrm{~m}$ Very Large Telescope's (VLT's) second unit telescope (UT2) was the first to reopen at the European Southern Observatory's (ESO's) Paranal Observatory, and science data began to flow again from the first instrument reactivated, the Ultraviolet and Visual Echelle Spectrograph (UVES). Although the choice of UVES to go first was based on technical considerations, it was also a fitting 20th birthday present, for UVES was first offered to the community in 2000, and has since been working incessantly, to the point that the ESO archive now contains UVES science spectra summing up to more than four years of total integration time. UVES is the second-oldest active VLT instrument, and through its entire career it has consistently led the ESO-wide publication metrics for the number of yearly papers published, only being surpassed by MUSE (Multi Unit Spectroscopic Explorer) in 2020.

UVES is a two-arm slit echelle spectrograph installed on the B Nasmyth platform of VLT-UT2 (Fig. 1). It was designed and built in-house at ESO and covers wavelengths between 300 and 1,100 $\mathrm{nm}$, of which $\sim 400 \mathrm{~nm}$ is simultaneously covered in two configurable ranges. It reaches a resolution $(R)$ of 110,000 in the red arm with a 0.3 " slit. With a 1 " slit, a one-hour exposure on a $V=17$ mag G0 star yields a signal-to-noise ratio of 27 at $400 \mathrm{~nm}(55$ at $600 \mathrm{~nm})$ per resolution element at $R=47,000$. UVES was designed from the beginning to be a workhorse, its design emphasizing versatility and efficiency. In addition to its own slit, a fibre link to the multi-object FLAMES (Fibre Large Array Multi Element Spectrograph) facility allows UVES to observe up to eight stars simultaneously. A continuously updated pipeline allows the extraction of science-ready data, and from 2013 UVES has been the first ESO instrument whose entire science archive is also made available in fully reduced form.

Over its long career, UVES has provided valuable contributions to many fields, from the measurement of fundamental constants, to the determination of primordial deuterium and lithium abundances, to the characterization of absorption systems in quasar sightlines, to the study of Solar System objects. It has been the instrument of choice for the study of the stellar populations in the Milky Way with the 'First Stars' Large Programme ${ }^{1}$ and the Gaia ESO public survey ${ }^{2}$, among others. With more than 2,200 refereed publications based on UVES data, it is impossible to list its achievements here, but this surely explains why ESO recently decided to keep UVES in operation for the foreseeable future.

However, in these two decades the instrumental landscape has changed: in Paranal alone, X-Shooter provides a larger wavelength coverage and higher efficiency, but lower resolution. ESPRESSO (Echelle Spectrograph for Rocky Exoplanets and Stable Spectroscopic Observations) possesses unparalleled resolution and stability, but does not reach the blue range that UVES covers. Upcoming facilities such as 4MOST (4-metre Multi-Object Spectroscopic Telescope) ${ }^{3}$ and MOONS (Multi-Object Optical and Near-infrared Spectrograph $)^{4}$ will offer extreme multiplexity but trade off on resolution and spectral coverage. The planned CUBES (Cassegrain U-band Efficient Spectrograph) will have extreme UV-blue efficiency, at the cost of lower resolution and no red coverage.

It is thus natural that, when ESO organized a virtual workshop to celebrate 20 years of UVES in October 2020, its focus was not to reminisce on the history of an exceptional instrument, but rather to answer the questions, what made UVES so successful for so long, and what to do to make it even better as it enters its third decade? The workshop community coalesced around a few outstanding points: efficiency and blue coverage, together with large spectral coverage, high resolution, and continuous availability to follow up on the expected steep increase in interesting targets provided by existing and upcoming survey facilities. Based on this input, solutions to maintain UVES as the state-of-the-art are now under consideration.

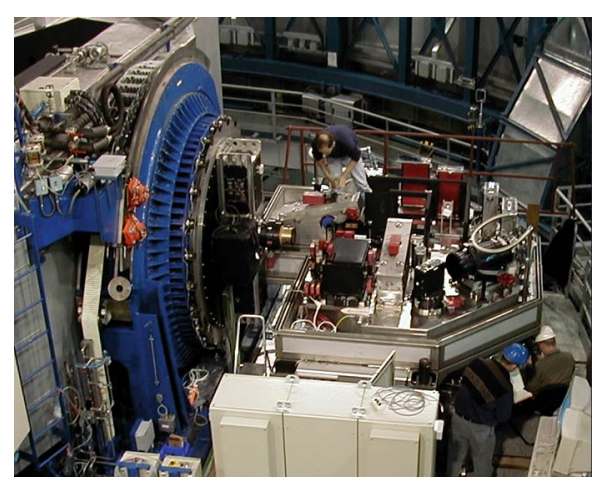

Fig. 1 | UVES being integrated on the Nasmyth platform of UT2 in 1999. The telescope altitude axis is on the left, painted blue. Credit: ESO.

The design of astronomical instruments is always an exercise in compromise. Specialized designs maximize specific performance metrics, and thus enable previously unfeasible science, but they come at a cost. General-purpose instruments such as UVES are not made obsolete by such developments: in fact, they make them viable, by covering for the needs the specialized design has to compromise against. But ultimately the reason for UVES's continued relevance is that it is an exceptionally well-designed and well-built instrument, and it was cared for by team after team of brilliant and dedicated engineers and astronomers. As the latest in this chain of caretakers, we are both impressed by its legacy and looking forward to its future.

\section{Luca Sbordone $\mathbb{D} \otimes$ and Camila Navarrete (D) $\square$ \\ European Southern Observatory, Santiago, Chile.

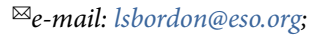 \\ Camila.Navarrete@eso.org}

Published online: 12 February 2021 https://doi.org/10.1038/s41550-021-01312-4

\footnotetext{
References

1. Cayrel, R. et al. Astron. Astrophys. 416, 1117-1138 (2004)

2. Gilmore, G. et al. The Messenger 147, 25-31 (2012).

3. de Jong, R. S. Nat. Astron. 3, 574 (2019).

4. Cirasuolo, M. et al. The Messenger 180, 10-17 (2020).
} 